\title{
Wakefields Generated by a Tightly Focused Laser Beam
}

\author{
D. F. Gordon, R. F. Hubbard, P. Sprangle, B. Hafizi \\ Naval Research Laboratory, Plasma Physics Division, Washington, DC 20375, USA
}

\begin{abstract}
Wakefields generated by a tightly focused laser beam $\left(w_{0} \ll \lambda_{p} \equiv 2 \pi c / \omega_{p}\right)$ are characterized by a region of total electron cavitation near the head of the laser pulse, followed by a highly localized region of high electron density. The accelerating field maximizes at a spot size significantly less than $\lambda_{p}$. The focusing fields are generally larger than the accelerating field, and exhibit complex phasing detrimental for accelerator applications. Modeling of these wakefields is facilitated by the ponderomotive guiding center algorithm [1].
\end{abstract}

\section{INTRODUCTION}

In one dimension (1D), the amplitude of the accelerating field driven by a laser pulse of length $\lambda_{p} / 2$ is approximately [2]

$$
\frac{E}{E_{0}} \approx \frac{1}{2} \frac{a_{0}^{2}}{\sqrt{1+a_{0}^{2} / 2}}
$$

where $a_{0}$ is the peak vector potential of the laser pulse normalized to $m c^{2} / e$ and $E_{0}$ is the cold wavebreaking field given by $m c \omega_{p} / e$. When laser power is limited, high accelerating gradients can only be obtained by tightly focusing the laser pulse. If the spot size $w_{0}$ becomes smaller than $\lambda_{p}$, non-ideal behavior will result.

In this paper we consider wakefields that might be driven by the $\mathrm{T}^{3}$ laser at the Naval Research Laboratory. The $\mathrm{T}^{3}$ laser, recently upgraded, provides $10 \mathrm{TW}$ of $1 \mu \mathrm{m}$ light in a $400 \mathrm{fs}$ pulse. The pulse length fixes the plasma density through the resonance condition $\tau_{L}=\lambda_{p} / 2$, which gives $n_{e} \approx 2 \times 10^{16} \mathrm{~cm}^{-3}$ and $\lambda_{p} \approx 240 \mu \mathrm{m}$. Here, $\tau_{L}$ is the full width at half maximum (FWHM) of the laser's temporal pulse shape and $n_{e}$ is density of free electrons. The current focusing optic is an $\mathrm{f} / 4.5$ paraboloid, which gives a spot size at best focus of $w_{0} \approx 7.5 \mu \mathrm{m}$. Supppose that 6 TW will be used to optically inject electrons via the LIPA scheme [3], while $4 \mathrm{TW}$ will be used to drive a wakefield. Then, the peak vector potential at the best focus of the 4 TW beam will be $a_{0} \approx 1.9$.

These parameters introduce two difficulties. First, the spot size is much less than the plasma wavelength. This violates the 1D approximation. Second, the Rayleigh length is on the order of the pulse length. This violates the quasistatic approximation [4].

\section{PONDEROMOTIVE GUIDING CENTER ALGORITHM}

Numerical simulations of an experiment like the one described above are difficult. Fluid simulations fail due to the violence of the density fluctuations involved. Quasi-static particle simulations [5] have been tried, but were found to give non-physical results [6]. Fully explicit particle-incell (PIC) simulations could be used in principal, but these would be extremely costly in three dimensions (3D), even on a massively parallel computer. Fully explicit PIC simulations in two dimensions can only be done in slab geometry.

The ponderomotive guiding center (PGC) algorithm is a solution which does not make the quasi-static approximation, yet does not require resolution of the optical frequency. The method has been described in detail elsewhere [1], so we give only an outline here.

The PGC algorithm presumes there is a large separation between the frequency of the wakefields and the frequency of the laser fields. The wakefields are computed using standard PIC techniques combined with the exact Maxwell equations. The laser fields are computed according to an envelope equation for the complex valued vector potential $a$ defined by

$$
a=\frac{\tilde{a}}{2} e^{i \omega(t-z)}+c c
$$

where we have taken $c=1$. Using the speed of light variables $\zeta=z-t$ and $\tau=t$, the envelope equation is

$$
\left(-2 i \omega_{0} \partial_{\tau}+2 \partial_{\tau \zeta}+\Delta_{T}\right) a=\left\langle\frac{n_{e}}{\gamma}\right\rangle a
$$

where $\omega_{0}$ is the laser frequency, $\Delta_{T}$ is the transverse Laplacian, $\gamma$ is the relativistic Lorentz factor, and we use a system of units where the plasma frequency and the magnitude of the electronic mass and charge are unity. Also,

$$
\left\langle\frac{n_{e}}{\gamma}\right\rangle=\frac{n_{e}}{\sqrt{1+P^{2}+|a|^{2} / 2}}
$$

Here, $P$ is the slowly varying part of the momentum. Also, rapidly varying terms in $n_{e}$ are henceforth ignored.

Once the fields are computed particles are pushed according to

$$
\partial_{t} \mathbf{P}=-(\mathbf{E}+\mathbf{v} \times \mathbf{B})-\left\langle\frac{1}{\gamma}\right\rangle \nabla \frac{|a|^{2}}{4}
$$

where $\mathbf{E}$ and $\mathbf{B}$ are the slowly varying electric and magnetic fields associated with the wake. Finally, the charge 
density and current density are deposited onto the numerical grid for the solution of Maxwell's equations, while the averaged relativistic mass is deposited onto the numerical grid for the solution of the envelope equation.

\section{SIMULATION RESULTS}

The code turboWAVE [1] has recently been extended to incorporate a 3D PGC algorithm. Figs. 1 and 2 show results from a turboWAVE simulation where a 4 TW, 400 fs FWHM, $1 \mu \mathrm{m}$ wavelength laser pulse was focused to a $7.5 \mu \mathrm{m}$ spot size at the edge of a $2 \times 10^{16} \mathrm{~cm}^{-3}$ plasma. Here, the FWHM is defined with respect to the laser amplitude. The grid was $256 \times 128 \times 128$ with a cell size of $.05 \times .025 \times .025$. The simulation was run on 32 nodes of an IBM SP3.

Fig. 1 shows the wake after the pulse has diffracted to a spot size of $11 \mu \mathrm{m}$. The blue area near $\zeta=300 \mu \mathrm{m}$ is a region of total electron cavitation. The small red area near $\zeta=200$ is an extremely intense region of electron concentration. The peak electron density there is about 13 times the ambient density. Because the density spike is localized in all three dimensions, the accelerating field is also steepened. This is distinct from the behavior in 1D where the electric field becomes a sawtooth wave as the density wave steepens. The maximum value of the accelerating field is about $.05 E_{0}$. The peak radial fields are about four times larger than the peak accelerating field.

Fig. 2 shows the wake after the pulse has diffracted to a spot size of $22 \mu \mathrm{m}$. Total electron cavitation is still exhibited around $\zeta \approx 300$. the density spike at $\zeta \approx 200$ is now even larger with a peak value 36 times the ambient density. Correspondingly, the accelerating field is more strongly peaked near the density spike with a maximum value of $0.34 E_{0}$. This field structure is probably not well suited for acceleration since the accelerating region is very small. Note also that the radial fields are about twice as large as the accelerating fields.

Finally, Fig. 3 shows a plot of peak accelerating field vs. propagation distance for both the $1 \mathrm{D}$ theory and the $3 \mathrm{D}$ simulation. Here, propagation distance is referenced from the point where the peak of the laser pulse is exactly at the plasma-vacuum interface. This point is also the point of best focus. As shown in the figure, the 3D simulations and the 1D theory predict opposite behavior. According to the 1D theory, the accelerating field increases as the laser approaches best focus. According to the 3D simulation, the accelerating field decreases as the laser aproaches best focus. Presumably, if the simulation were continued the two curves would converge as the spot size became large. This implies that in 3D an optimum spot size exists. Two-dimensional simulations have indeed revealed an optimum spot size of about $35 \mu \mathrm{m}$. However, in the two dimensional simulations the spike in the electron density was much smaller.
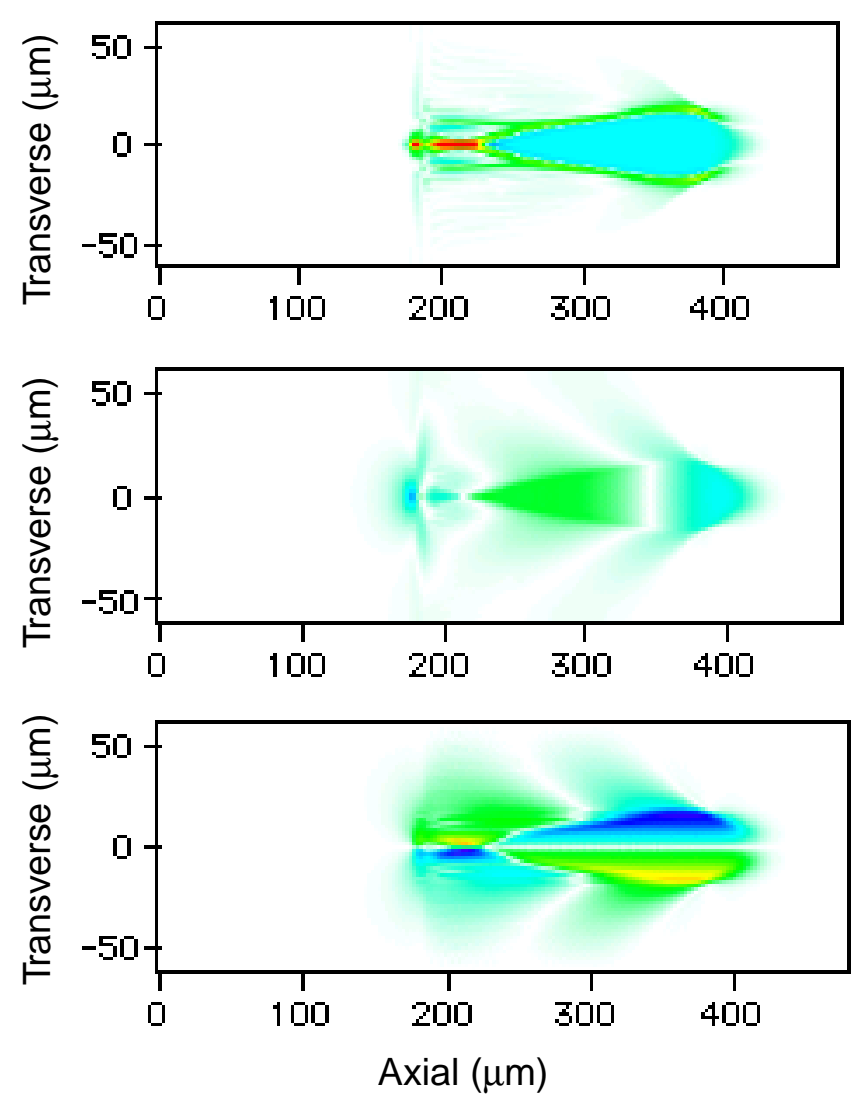

Figure 1: 3D simulation of the wakefield generated by a tightly focused multi-terawatt laser pulse after $1 z_{R}$. (a) charge density $\rho(\zeta, r)$ (b) axial electric field $E_{z}(\zeta, r)$ (c) radial electric field $E_{r}(\zeta, r)$

\section{CONCLUSIONS}

The three-dimensional ponderomotive guiding center algorithm can be used to study the wakefields generated by a tightly focused laser. Preliminary results indicate that such interactions are dominated by total electron cavitation followed by a dramatic radial collapse of the electrons. This leads to an intense density spike localized in all three dimensions. The field structure does not appear to be well suited for acceleration since the strongest fields are highly localized. The radial fields are also very large which is likely to have an adverse effect on the emittance of the output beam. This issue will be examined more closely by including test particles in the simulation.

\section{ACKNOWLEDGEMENTS}

This work was supported by the Department of Energy and the Office of Naval Research. This work was performed while D.F. Gordon held a National Research Council Research Associateship. 

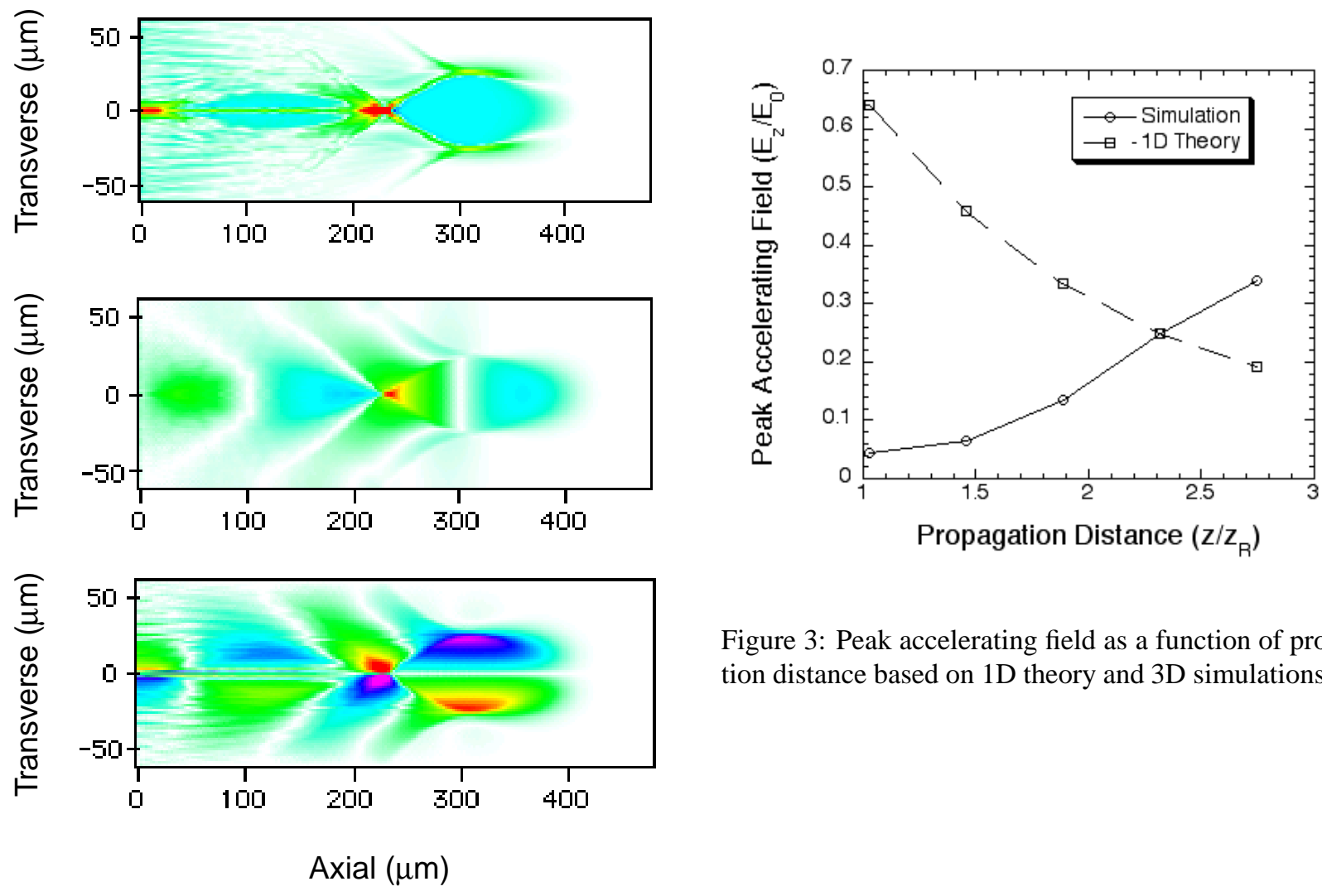

Figure 3: Peak accelerating field as a function of propagation distance based on 1D theory and 3D simulations

Figure 2: 3D simulation of the wakefield generated by a tightly focused multi-terawatt laser pulse after $2.7 z_{R}$. (a) charge density $\rho(\zeta, r)$ (b) axial electric field $E_{z}(\zeta, r)$ (c) radial electric field $E_{r}(\zeta, r)$

\section{REFERENCES}

[1] D. F. Gordon, W. B. Mori, and T. M. Antonsen, Jr., IEEE Trans. on Plasma. Sci. 28, 1224 (2000).

[2] E. Esarey, P. Sprangle, J. Krall, and A. Ting, IEEE Trans. on Plasma Sci. 24, 252 (1996).

[3] C. I. Moore et al., Phys. Rev. Lett. 82, 1688 (1999).

[4] P. Sprangle et al., Phys. Rev. Lett. 69, 2200 (1992).

[5] P. Mora and T. Antonsen, Phys. Plasmas 4, 217 (1997).

[6] R. F. Hubbard, private communication. 